\title{
THE DEVELOPMENT OF A REMOTE LABORATORY FOR INTERNET-BASED ENGINEERING EDUCATION
}

\author{
Ahmed Chiheb Ammari \\ Institut National des Sciences Appliquées et de Technologie (INSAT) \\ Unité de Recherche en Matériaux Mesures et Applications (MMA) \\ Jaleleddine Ben Hadj Slama \\ Institut National des Sciences Appliquées et de Technologie (INSAT) \\ Laboratoire des Systèmes Électriques (LSE), École Nationale d’Ingénieurs de Tunis (ENIT)
}

\begin{abstract}
The development of feasible and cost-effective remote engineering and science laboratories is one of the most important problems facing the progress of online technical education. In this paper, we describe the development of a complete remote laboratory for the instruction of control engineering. Equipped with common industrial sensors and actuators, the system represents temperature and flow processes regulated with an industrial Programmable Logic Controller (PLC). Using local monitoring, we enable students to perform interactive real plant experimentation in control and automation without the overhead incurred in maintaining a full presence laboratory.
\end{abstract}

\section{KEYWORDS}

Institutional Transformation, Leadership, Strategic Planning, Community, Scalability

\section{INTRODUCTION}

Laboratory experiments are vital components of the teaching of science and engineering [1]. They allow the application and testing of theoretical knowledge in practical learning situations [2]. In a full presence laboratory, students operate real devices and manipulate and measure real objects while located in the same space as the laboratory equipment. However, current scientific practice demands that institutions buy and maintain expensive laboratory instrumentation, and many schools, facing budget pressures, are finding it difficult to provide students with adequate laboratory time. Nowadays, Internet-based techniques are frequently used for setting up remote laboratory access. Remote control of real experiments in the science classroom has the potential to reduce costs, increase time-efficient use of facilities, and ensure laboratory access for students [3, 4].

Many laboratory experiments conducted by remote, online methods have been reported in the literature [5]. Our earliest examples of such systems were described in 2002 [6, 7]. Salzmann [8] described an Internet-accessible servo device and how it can be used to assist student learning. Del Alamo [9] proposed similar arrangements in which students can remotely test semiconductor devices in web-enabled experiments. Trevelyan [10] showed how a telerobot project built at the University of Western Australia can provide a useful learning environment for mechatronics studies.

In a complete e-learning platform, remote laboratories have to merge into global and homogeneous 
learning management systems (LMS) [11]. One main goal of an LMS is to manage learning resources in a platform-independent environment while providing access to experimentation sessions from a uniform elearning context. Research has recently appeared on the topic of integrating a remote laboratory into a global LMS. Benmohamed [12] proposed a generic framework to allow instructors to integrate remote laboratories into their LMS and enable creation, distribution, and exchange of pedagogical scenarios for practical works. Kolberg [13] showed how web services can be used to enable laboratories' integration with modern LMS.

In this paper we describe the building of a remote linear control engineering laboratory for integration in a global, homogeneous LMS environment. In control engineering education, students should attain knowledge and skills pertaining to control systems modeling in order to develop controllers that enforce performance requirements [14]. After a controller is designed and implemented, observation of the resulting dynamics gives valuable insight into design concepts.

The objective of the laboratory we have developed is to offer students interactive real-world plant experimentation in control and automation, problem solving, data gathering, and scientific interpretation. With Internet access, we enable students to perform tests with system security and without the overhead incurred in maintaining a full presence laboratory.

\section{THE PHYSICAL SYSTEM}

The system is a platform for use in linear control engineering and automation experiments. On a smaller scale, it replicates PLC-controlled temperature and flow processes equivalent to those implemented in biochemical industries. A synoptic scheme of the system is shown in Figure 1.

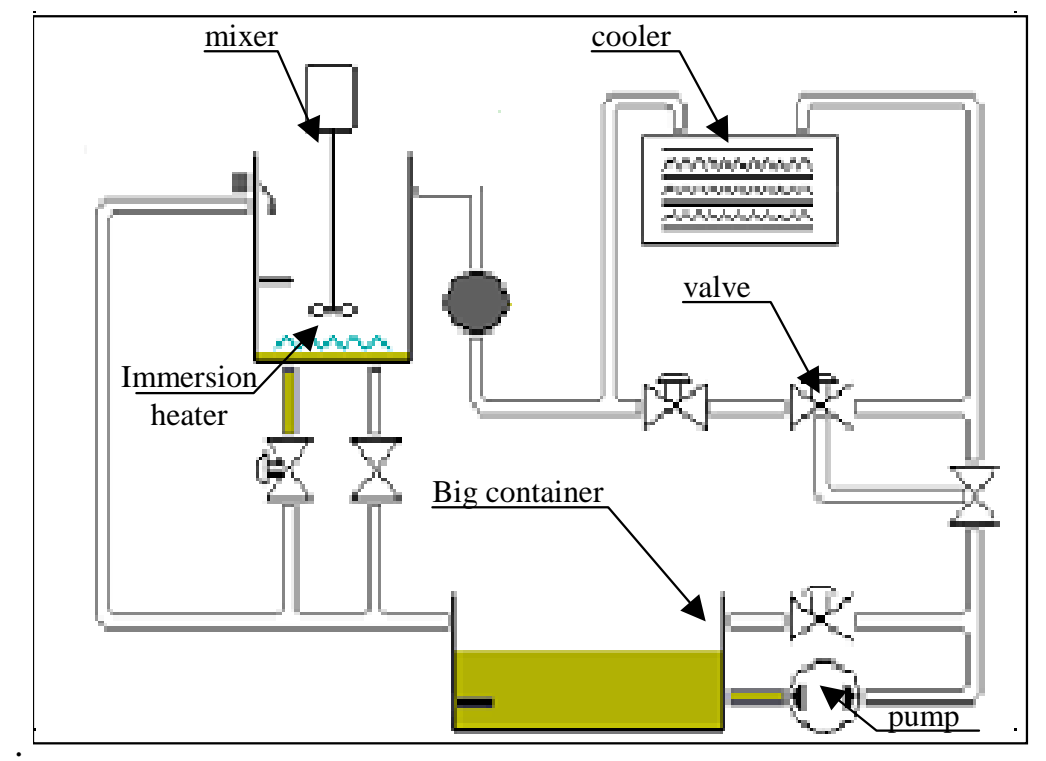

Figure 1. Synoptic Scheme of the System

The process is constituted by a main container that can be filled with a liquid. This liquid, pumped with an AC pump, may be driven directly or through a cooling system to a jar. An industrial electronic speed controller drives the AC pump. Within the jar is placed an immersion heater and a mixer system for temperature homogenization. The liquid may also be drained directly from the jar to the big container. 
The circulation path of the liquid along all circuit tubing may be modified using electrical or manual valves selected for industrial applicability. Manual valves are used to introduce a system perturbation or to reproduce industrial realities. The system is also equipped with all the sensors necessary for measuring key parameters, as described below:

- Three Pt100 temperature sensors fixed respectively on the big container, the tubing, and the jar.

- One turbine flow liquid sensor mounted on the tubing

- Two level sensors, one placed on the big container and one on the jar.

A low-cost CPM2A PLC (Omron Electronics) controls the process. The system parameters are fixed manually using a dedicated console interface directly connected to one of the PLC serial ports. A second serial port will be connected to a personal computer running a dedicated monitoring application. All the controlled parameters are directly displayed on the system board using specific digital displays. A photograph of this system is shown in Figure 2.

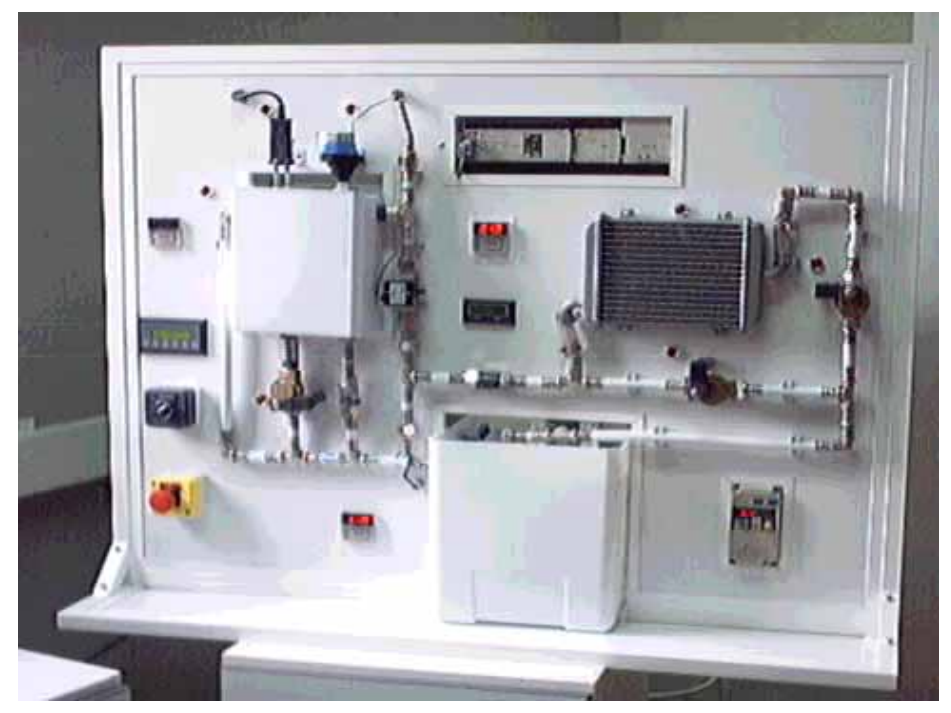

Figure 2. A Photograph of the System

So far, the developed system may be used to address the following educational goals:

- Testing of and experimentation with common sensors and actuators used in biochemical industrial processes

- Programming an off-the-shelf CPM2A PLC using industrial programming tools provided by Omron

- Proportional-integral-derivative (PID) manual control of temperature and flow processes using an industrial interface PLC console

For linear control experiments, system model analysis, parameter identification, and PID performance evaluations, a dedicated local monitoring application is first developed. This will be presented in the next section. 


\section{LOCAL MONITORING}

For local monitoring, the second RS232C communication port of the PLC is used to establish a serial connection with a local computer. Exchange of data between the PLC and the computer is performed according to a specific Omron proprietary conversational protocol [15]. The computer prepares a request by building a specific command and sends it to the PLC across the serial line. The PLC prepares the response, maps it in a sort of subroutine, and sends it back to the requesting master computer. A specific program in this computer decodes the received subroutine to extract information related to the specific request.

To ensure total control of the code of the monitoring application, the Omron proprietary command/response protocol was first analyzed and then implemented. A dedicated communication module was also developed. We used a local computer running this protocol-decoding module to interface with the PLC monitor all its input/output states, and fix outputs to desired values. Figure 3 shows a simple diagram of the interface.

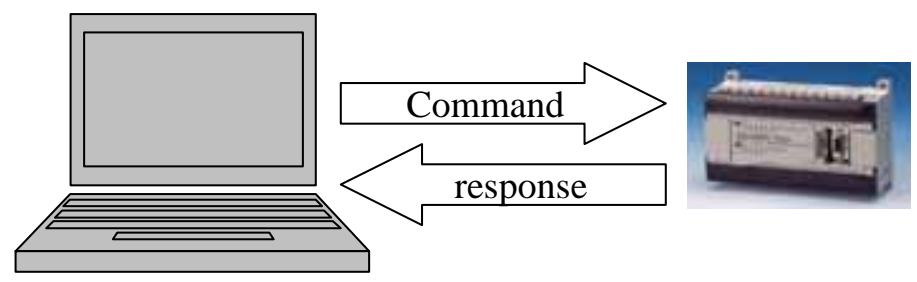

Figure 3. The Conversational Protocol

The output of the communication module is used to animate supervisory interfaces of the process with a refresh rate of about 100 milliseconds. All the interface pages and communication modules were implemented from scratch using Microsoft Visual Basic 6. The synoptic supervisory interface module was developed to enable the user to view the state of all actuators and sensors and to control each output element separately. An example of such a synoptic page is shown in Figure 4. In this figure, the liquid flow and the temperature values on the container, jar, and tubing are clearly indicated. Button animations are used to indicate the state of all the valve elements on the system.

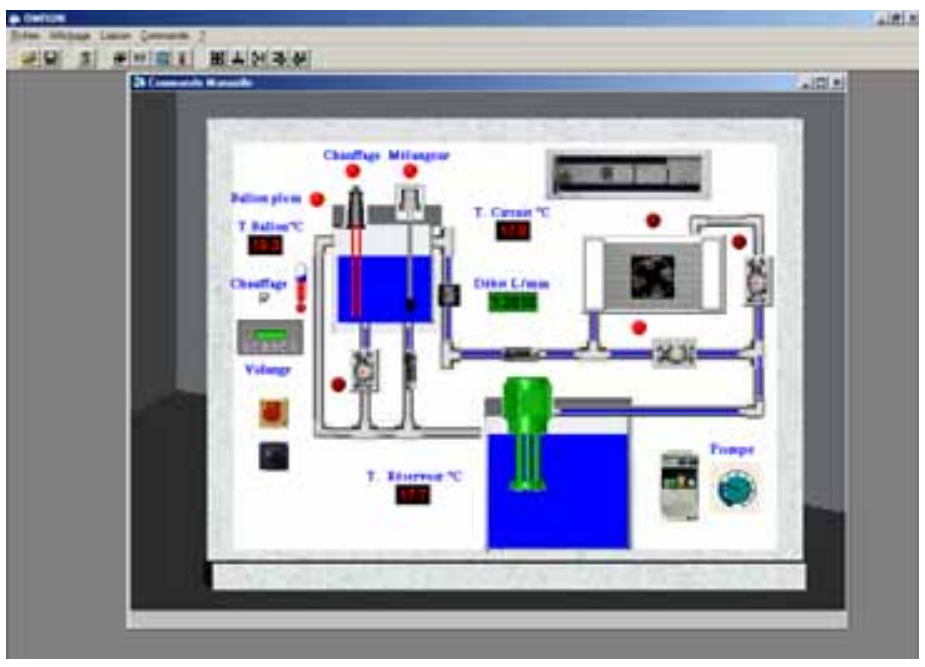

Figure 4. The Synoptic Supervisory Interface 
An open-loop control module was also developed for identification testing of the temperature and flow systems. Open-loop control allows step, ramp, or sine inputs to the system. The response associated with the excitation function is plotted and may be stored as an Excel file for further use in MATLAB. An example of an open-loop system response is shown in Figure 5. In this figure, an open-loop response of the liquid flow process in response to a step input is shown.

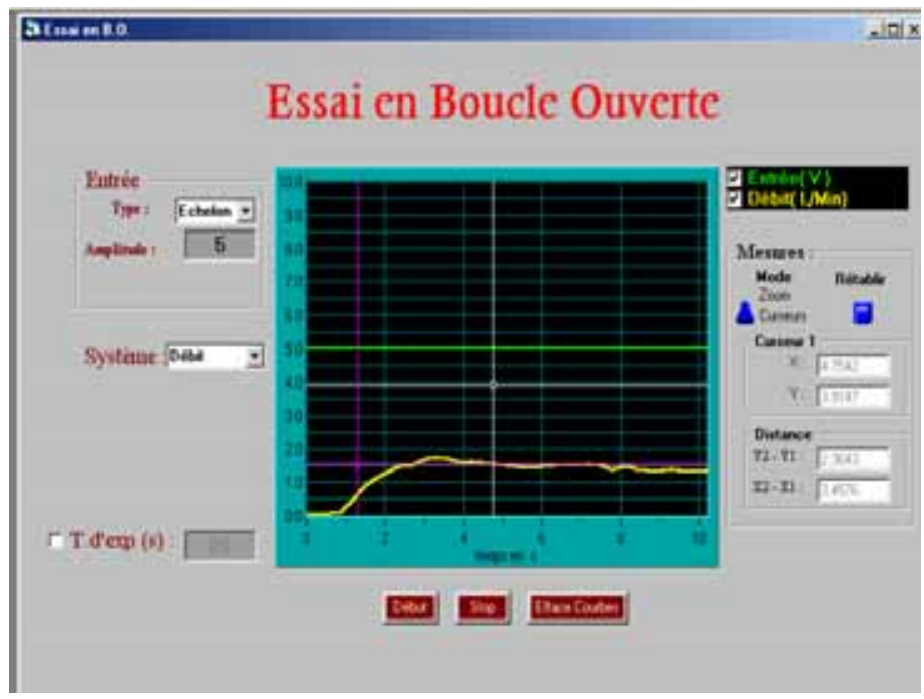

Figure 5. Open-Loop Control Form

In the next part of the laboratory implementation, students design PID parameters for the temperature and flow controllers. To evaluate the controllers' performance, we developed dedicated modules and interfaces (Figures 6 and 7). For example, Figure 6 presents a proportional-integral controlled closed-loop response of the flow process (the derivative $D$ parameter was not used for that case). For such modules, the sampling period is an input parameter. The response may also be stored as an Excel file for further analysis.

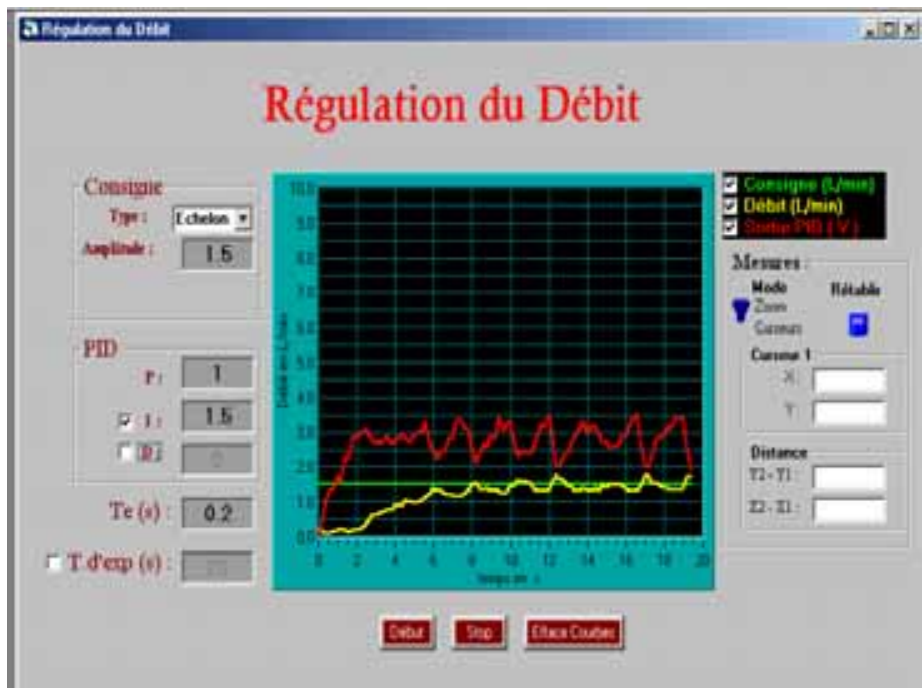

Figure 6. PID Flow Control 
With the local monitoring application, the system can be used for practical laboratory experiments in control engineering, particularly for temperature and flow processes. Among the possibilities are the following:

- Identification test experimentation of temperature and flow processes

- Systems analyzing and modeling using knowledge and skills from control system theory

- Design of controllers that enforce performance requirements

- Systems simulations with MATLAB/SIMULINK simulators

- $\quad$ Systems experiments for performance evaluations

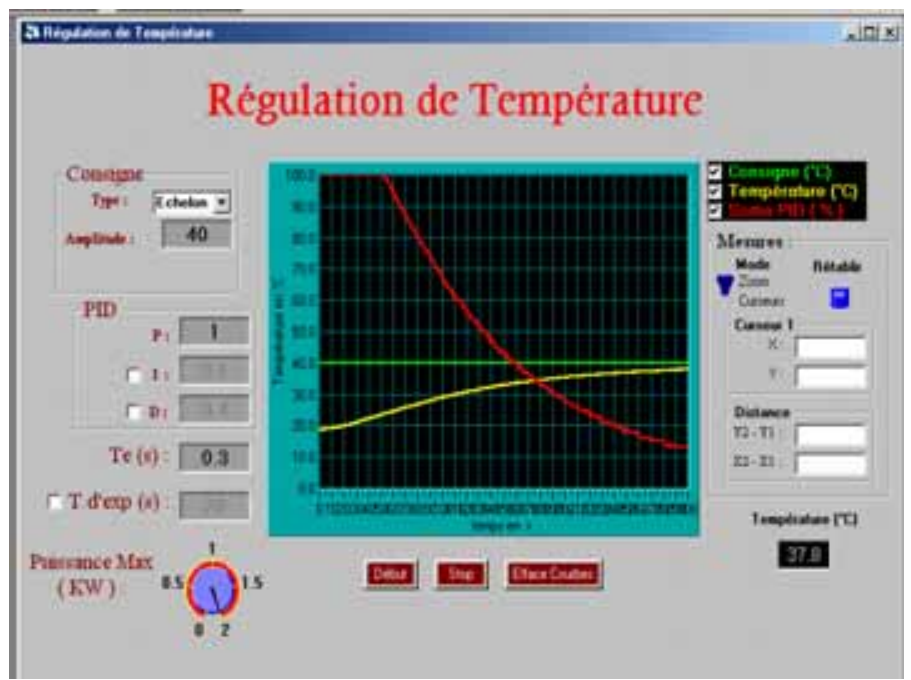

Figure 7. PID Temperature Control

\section{CLIENT/SERVER APPLICATION}

To enable use of the system over a network, we selected a client/server architecture as described in [16]. This architecture is presented in Figure 8.

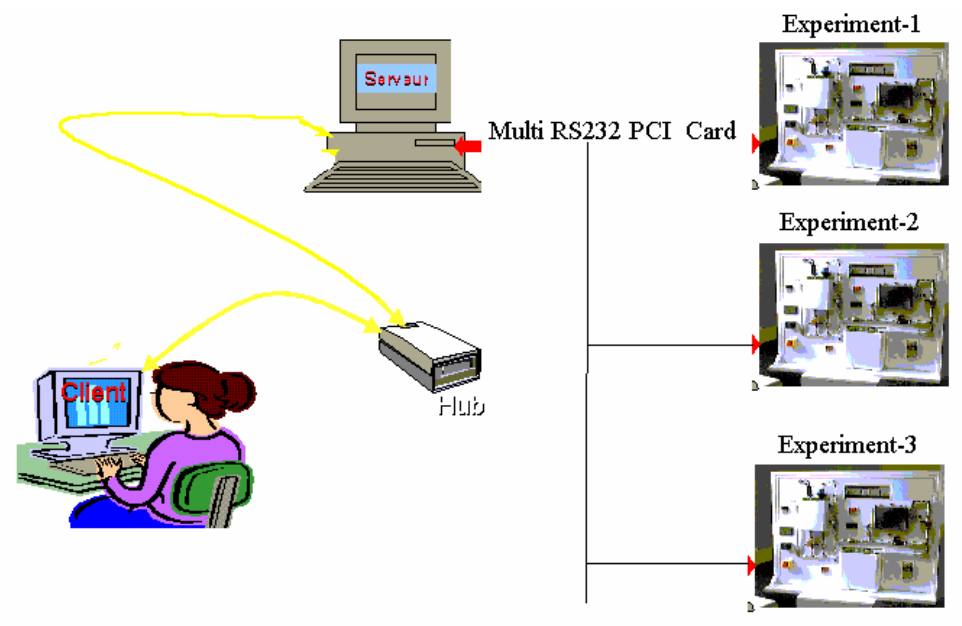

Figure 8. Multi-Platform Client/Server Architecture 
The server is connected to the system through an RS232 line. A server application acts as a gateway between the system and the TCP/IP network. Typically, the server is always listening to clients in order to make connections. After a connection is made, the client makes a request to the server. The server transmits the request as a command to the PLC and then receives and decodes responses, responding to the client with the requested data or an error message. The conversation continues until one side-usually the client-decides it wants to disconnect, at which time the session is terminated.

For security reasons and because there is currently only one prototype platform, no more than one client can be connected to the system at any one time. In a client/server model, a single server often services requests from many clients. For our first server application, if a client gets connected, the server will refuse connection to further clients until the first is disconnected.

Given the success of the first prototype, two other platforms are under development. To address the scalability limitations of the first server application, we equipped the PC running the server with a PCI card of four RS232 ports as presented in Figure 8, and we integrated a configuration module into the server application. This module lets the administrator configure the number of platforms used and the associated RS232 ID ports. Therefore, if a client gets connected, the server uses a sort of lookup table to select a free unused platform. If none are available, it will tell the client to reconnect later.

On the client side, applications are being implemented as ActiveX controls. An ActiveX control is an object that can be placed in a form to enable or enhance a user's interaction with an application. ActiveX controls can be incorporated into other controls or embedded into Web HTML pages. When an ActiveX control embedded in a HTML document on the Web is accessed, the control is first downloaded and registered in the client computer, and then run with a standard Web browser. Thus, anyone with a standard Web browser and a valid password can execute our client applications to interact with the system.

Four ActiveX control modules have been implemented. First, the synoptic supervisory control module enables the user, using a standard web browser, to view the state of all actuators and sensors and to control each output element separately. For identification test purposes of the temperature and the flow systems, an open-loop ActiveX control module is also being developed. Test result responses may be saved as an Excel file on the client computer for further use.

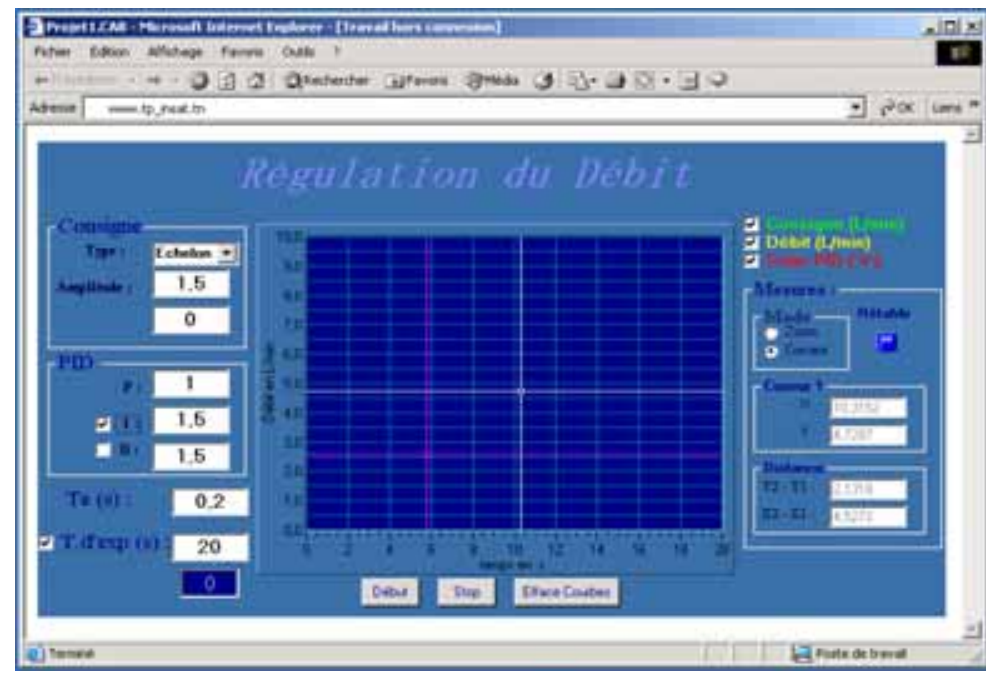

Figure 9. The ActiveX PID Flow Control Module Running with an Internet Explorer Web Browser 
For PID controller synthesis and laboratory test experiment evaluations, dedicated ActiveX modules are also being developed. An example of a PID flow control module is shown in Figure 9. In this figure, we have the PID flow control form presented in Section II implemented as an ActiveX and running on the client computer using a Web browser.

\section{CONNECTING TO THE WEB}

For distance learning and remote laboratory test purposes, we developed a dedicated dynamic website using an Apache web server, PHP, and MySql. PHP is an open source scripting language; PHP commands embedded in HTML Web pages are executed in the web server to generate dynamic pages. MySql is an open source relational database management system using Structured Query Language (SQL) for adding, accessing, and processing data in the database.

Using these techniques and resources, we developed pages to secure access and use of the system. Thus, a student asking for a connection has first to register for an appointment. An administrator manages all appointment requests. The user is notified of the acceptance or denial of his request automatically by email. If the request is accepted, the user has to log on to the system at to the given appointment time and is given one hour to finish his test experiments and retrieve results. Given the system dynamics, it is estimated that one hour is sufficient to complete experiments. However, if an experiment is under test and time expires, the server lets this experiment finish and then stores results in an Excel file. It will not be possible for this client to perform further commands; he is authorized only to retrieve the last experiment results.

As indicated above, with only one platform, no more than one person can be connected at a time. For future use, it will be possible with a single server configuration to authorize multiple client connections according to the number of connected platforms. An example of logon and identification pages is given in Figure 10.

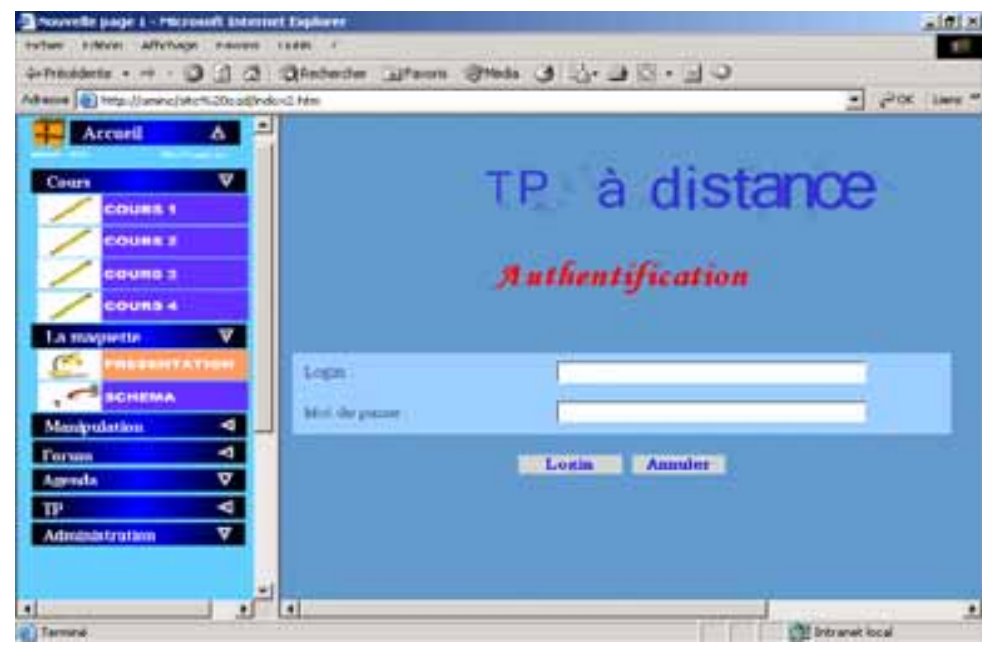

Figure 10. The Client Identification Page

For administration and maintenance purposes, dedicated web pages have been developed for users and system administration. Figure 11 presents an example of the administration menu, which displays submenus for users and system administrators, and for the historic use of the system. The menu also contains a link to a dedicated forum to enable users to exchange ideas and messages concerning system practice. 


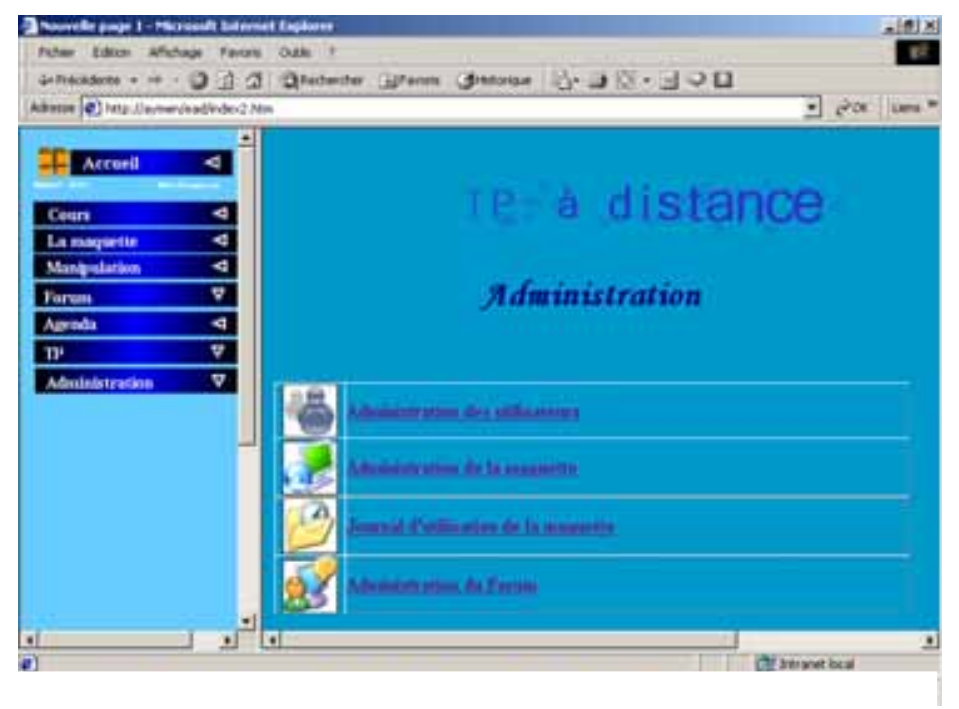

Figure 11. The Administration Menu

Finally, using a classic navigating tool and an Internet connection, students far from the university, at home or in cyberspace, may be connected to the website through the university web server. After registering, they $\log$ on to the system at a previously appointed time and have one hour to finish experiments, retrieve and print results, and observe the curves representing the evolution of the process according to given parameters. They may also profit from the dedicated laboratory forum.

This remote laboratory has been beta-tested at the National Institute of Applied Sciences and Technology (INSAT) in Tunisia. The results of these trials and the level of user acceptance of the system are promising. However, some problems appeared due to the lack of administrative support and the need for collaborating users to be able to work together. These problems can be overcome with some modifications in the present system architecture to enable integration of this laboratory within the existing e-learning environment of the Virtual University of Tunis. To enable this integration, we are currently working on an approach based on web services technology as described in [13].

\section{CONCLUSIONS}

In this paper, we describe the structure and function of a remote linear control engineering laboratory developed to offer students interactive real-world plant experimentation in control and automation. The system represents industrial PLC-controlled temperature and flow processes. First, the physical system was developed with all necessary industrial sensors and actuators. For local monitoring of test experiments, the Omron PLC protocol was implemented, and dedicated experimentation modules and interface forms were developed using Microsoft Visual Basic 6. To enable use of this laboratory over a network, we selected a client/server architecture, and all client and server software modules were implemented. For long-distance learning purposes, we developed a dynamic website and a dedicated forum. Our approach was based on PHP scripting language to support access to experiments via a web server, and ActiveX controls to visualize results in the client computer. For better utilization, this laboratory needs to be integrated within a global and homogeneous learning management system (LMS).

\section{ACKNOWLEDGEMENTS}

The authors gratefully acknowledge the contributions of Y. Dorgham, M. Karray, M. Mlouka, M. A. 
Ismail, A. Hafsaoui, and M. A. Iben Abdelhamid.

\section{REFERENCES}

1. Gillet, D., F. Geoffroy, K. Zeramdini, A. V. Nguyen, Y. Rekik and Y. Piguet. The Cockpit: An Effective Metaphor for Web-based Experimentation in Engineering Education. International Journal of Engineering Education 19(3): 389-397, 2003.

2. Law, L. C. and A. Babich. Web-based Learning with a Virtual Simulation Lab: Experience and Evaluation. Proceedings of the 5th International Conference on New Educational Environments (ICNEE), Lucerne, Switzerland, 26-28 May 2003.

3. Zimmer, T., D. Geoffroy, M. Billaud and Y. Danto. The eLab For Electrical Engineering Education. Proceedings of the IEEE 4th International Conference on Information Technology Based Higher Education and Training (ITHET03), Marrakech, Morocco, July 7-9 2003.

4. Lelevé, A., H. Benmohamed, P. Prevot and C. Meyer. Remote Laboratory Towards an Integrated Training System. Proceedings of the IEEE 4th International Conference on Information Technology Based Higher Education and Training (ITHET03), Marrakech, Morocco, July 7-9 2003.

5. Böhne, A., N. Faltin and B. Wagner. Synchronous TeleTutorial Support in a Remote Laboratory for Process Control. In: W. Aung, R. Altenkirch, T. Cermak, R. W. King, L. M. S. Ruiz (eds.), INNOVATIONS 2004: World Innovations in Engineering Education and Research, International Network for Engineering Education and Research (INEER), 2004.

6. Ben Hadj Slama, J., A. C. Ammari and M. Karray. Supervision à distance des precessus industriels. Proceedings of the CRATT'2002 1: 170-177, December 2002.

7. Ammari, A. C., J. Ben Hadj Slama and R. Ajroudi. Supervision et contrôle d'un système de contrôle en réseau par Internet. Proceedings of the CRATT'2002 1: 178-185, December 2002.

8. Salzmann, C., D. Gillet and P. Huguenin. Introduction to Real-Time Control Using LabView with an Application to Distance Learning. International Journal of Engineering Education 16(2): 2000.

9. Del Alamo, J., L. Brooks, C. McClean, J. Hardison, G. Mishuris, V. Chang and L.Hui. A Webenabled Remote Laboratory for Microelectronic Device Characterization. Proceedings of the 2002 Network Learning Conference (NL2002), Berlin, Germany, May 2002.

10. Trevelyan, J. Remote Laboratories and Team Skills in Mechatronics. Mechatronics and Machine Vision in Practice Conference, Perth, Western Australia, December 2003. http://telerobot.mech.uwa.edu.au/Information/Trevelyan-M2VIP2003.pdf.

11. Kolberg, S. and T. A. Fjeldly. Integration of Remote Laboratory Exercises into Learning Management Systems. IASTED International Conference on Education \& Technology 2005 (ICET 2005), Calgary, Alberta, Canada, July 4-6, 2005.

12. Benmohamed, H., A. Lelevé and P. Prévot. Generic Framework for Remote Laboratory Integration. Proceedings of the IEEE International Conference on Information Technology Based Higher Education and Training (ITHET), Santo Domingo, Dominican Republic, July 7-9, 2005.

13. Kolberg, S. and T. A. Fjeldly. Remote Educational Laboratory System Based on Web Services Standards. In: W. Aung, R. W. King, J. Moscinski, S. H. Ou, L. M. S. Ruiz (Eds.), INNOVATIONS 2005: World Innovations in Engineering Education and Research, International Network for Engineering Education and Research (INEER), 2005.

14. Ogata, K. Modern Control Engineering, 3rd edition. London, UK: Prentice-Hall International Ltd, 1998.

15. Held, G. Understanding Data Communications: From Fundamentals to Networking, 3rd edition. West Sussex, UK: John Wiley \& Sons Ltd, 2000.

16. Esche, S. K., C. Chassapis, J. W. Nazalewicz and D. J. Hromin. An Architecture for Multi-user Remote Laboratories. World Transactions on Engineering and Technology Education 2(1): 2003. 


\section{ABOUT THE AUTHORS}

Ahmed Chiheb Ammari received an engineering degree from the National School of Engineering in Monastir, Tunisia, in 1993, and a Ph.D. degree in electrical engineering from the National Polytechnic Institute of Grenoble (INPG) in France in 1996. Since 1997, he has been an assistant professor of electrical engineering at the Institut National des Sciences Appliquées et de Technologies (INSAT) in Tunis, Tunisia. His research interests include power systems and electrical synchronous machines with unbalanced state analysis. He is currently interested in remote laboratories and Internet-based applications, real-time embedded control, and MPSoC codesign.

Jaleleddine Ben Hadj Slama received his engineering degree and Ph.D. degree in electrical engineering from the École Centrale de Lyon, Lyon, France, in July 1994 and December 1998 respectively. Since 1998, he has been an assistant professor of electrical engineering at the Institut National des Sciences Appliquées et de Technologies (INSAT) in Tunis, Tunisia. His research interests include electromagnetic compatibility studies in power electronics, numerical methods, monitoring of industrial processes, and remote electrical engineering laboratories. 